\title{
The radio SNR G65.1+0.6 and its associated pulsar J1957+2831
}

\author{
W. W. Tian ${ }^{1,2}$ and D. A. Leahy ${ }^{2}$ \\ 1 National Astronomical Observatories, CAS, Beijing 100012, PR China \\ 2 Department of Physics \& Astronomy, University of Calgary, Calgary, Alberta T2N 1N4, Canada
}

Received 3 March 2006 / Accepted 18 May 2006

\section{ABSTRACT}

\begin{abstract}
New images of the radio Supernova Remnant (SNR) G65.1+0.6 are presented, based on the $408 \mathrm{MHz}$ and $1420 \mathrm{MHz}$ continuum emission and the HI-line emission data of the Canadian Galactic Plane Survey (CGPS). A large shell-like structure seen in the $2695 \mathrm{MHz}$ Effelsberg map appears to have nonthermal spectral index. HI observations show structures associated with the SNR G65.1+0.6 in the radial velocity range of -20 to $-26 \mathrm{~km} \mathrm{~s}^{-1}$ and suggest a distance of $9.2 \mathrm{kpc}$ for the SNR. The estimated Sedov age for G65.1+0.6 is $4-14 \times 10^{4}$ yr. The pulsar (PSR) J1957+2831 is possibly associated with G65.1+0.6, with consistent distance and kinematic age estimate, but different characteristic age than the SNR. The EGRET source 3EG J1958+2909 and $\gamma$-ray source 2CG 065+00 are also near the eastern edge of the SNR but do not agree in position with the pulsar and are likely not associated with the SNR. The SNR's flux densities at $408 \mathrm{MHz}(8.6 \pm 0.8 \mathrm{Jy}), 1420 \mathrm{MHz}(4.9 \pm 0.5 \mathrm{Jy})$ and $2695 \mathrm{MHz}(3.3 \pm 0.5 \mathrm{Jy})$ have been corrected for flux densities from compact sources within the SNR. The integrated flux density based spectral index $\left(S_{v} \propto v^{-\alpha}\right)$ between $1420 \mathrm{MHz}$ and $408 \mathrm{MHz}$ is $0.45 \pm 0.11$ and agrees with the T-T plot spectral index of $0.34 \pm 0.20$. The nearby SNR DA495 has a T-T plot spectral index of $0.50 \pm 0.01$.
\end{abstract}

Key words. ISM: general - radio line: ISM - radio continuum: galaxies

\section{Introduction}

Since G65.1+0.6 was discovered as a low surface brightness SNR (Landecker et al. 1990), little research on this SNR has been done. Due to lack of independent distance and age estimates for G65.1+0.6, it was difficult for previous authors to compare the SNR with nearby discovered pulsars (Lorimer et al. 1998). The detections of an EGRET source 3EG J1958+2909 (Hartman et al. 1999) and a TeV $\gamma$-ray source 2CG 065+00 (Alexandreas et al. 1991; Swanenburg et al. 1981) which partly overlap the SNR G65.1+0.6 give increased reason to further study G65.1+0.6. In this paper, we present the SNR's continuum images at higher sensitivity and resolution than previously at $408 \mathrm{MHz}$ and $1420 \mathrm{MHz}$, and first investigate HI-line emission at various radial velocities for detecting interactions of the remnant with the surrounding gas, estimate its distance and age, and access its possible association with the nearby EGRET source, $\gamma$-ray source and pulsar. We see evidence for a large SNR-like shell in the $2695 \mathrm{MHz}$ Effelsberg map, and investigate the evidence below.

\section{Observations and analysis}

The continuum and HI emission data sets come from the CGPS, which is described in detail by Taylor et al. (2003). The data sets are mainly based on observations from the Synthesis Telescope (ST) of the Dominion Radio Astrophysical Observatory (DRAO). The spatial resolution of the continuum images of G65.1+0.6 is $0.8^{\prime} \times 1.7^{\prime}$ at $1420 \mathrm{MHz}$ and $2.8^{\prime} \times 5.9^{\prime}$ at $408 \mathrm{MHz}$. The synthesized beam for the HI line images is $58^{\prime} \times$ $2.0^{\prime}$ and the radial velocity resolution is $1.32 \mathrm{~km} \mathrm{~s}^{-1}$. DRAO ST observations are not sensitive to structures larger than an angular size scale of about $3.3^{\circ}$ at $408 \mathrm{MHz}$ and $56^{\prime}$ at $1420 \mathrm{MHz}$. Thus the CGPS includes data from the $408 \mathrm{MHz}$ all-sky survey of Haslam et al. (1982), sensitive to structure greater than $51^{\prime}$, and the Effelsberg $1.4 \mathrm{GHz}$ Galactic plane survey of Reich et al. (1990, 1997), sensitive to structure with resolution $9.4^{\prime}$ for large scale emission (the single-dish data are freely available by http://wwW .mpifr-bonn.mpg.de/survey.html). The loworder spacing HI data is from the single-antenna survey of the CGPS area (Higgs \& Tapping 2000) with resolution of 36'. See Taylor et al. (2003) for detail of the method of combining the synthesis telescopes and single dish observations.

We analyze the continuum and HI images of G65.1+0.6 and determine its flux densities and distance. For G65.1+0.6, integrated flux density's errors are found by comparing results for several different choices of background region. For compact sources, the flux density's errors are taken as the formal Gaussian fit errors. The influence of compact sources within the SNR is much reduced by employing similar methods to Tian \& Leahy (2005).

\section{Results}

\subsection{Continuum emission}

The CGPS continuum images at $408 \mathrm{MHz}$ and $1420 \mathrm{MHz}$ are shown in the upper left and right panels of Fig. 1. The 6 boxes labeled with letters A-F and used for T-T plots are shown in the left. The upper right of Fig. 1 shows positions and error circles of EGRET source 3EG J1958+2909 (marked by number 1 ) and $\gamma$-ray 2CG $065+00$ (marked by number 2 ). Pulsar J1957+2831 is marked by letter X. The lower left shows the $1420 \mathrm{MHz}$ map convolved to the same resolution as the $408 \mathrm{MHz}$ map. The $2695 \mathrm{MHz}$ Effelsberg map is reproduced in the lower right for reference (Fürst et al. 1990). The Effelsberg map has a resolution of $4.3^{\prime}$ and a sensitivity of $50 \mathrm{mK} T_{\mathrm{B}}$. 

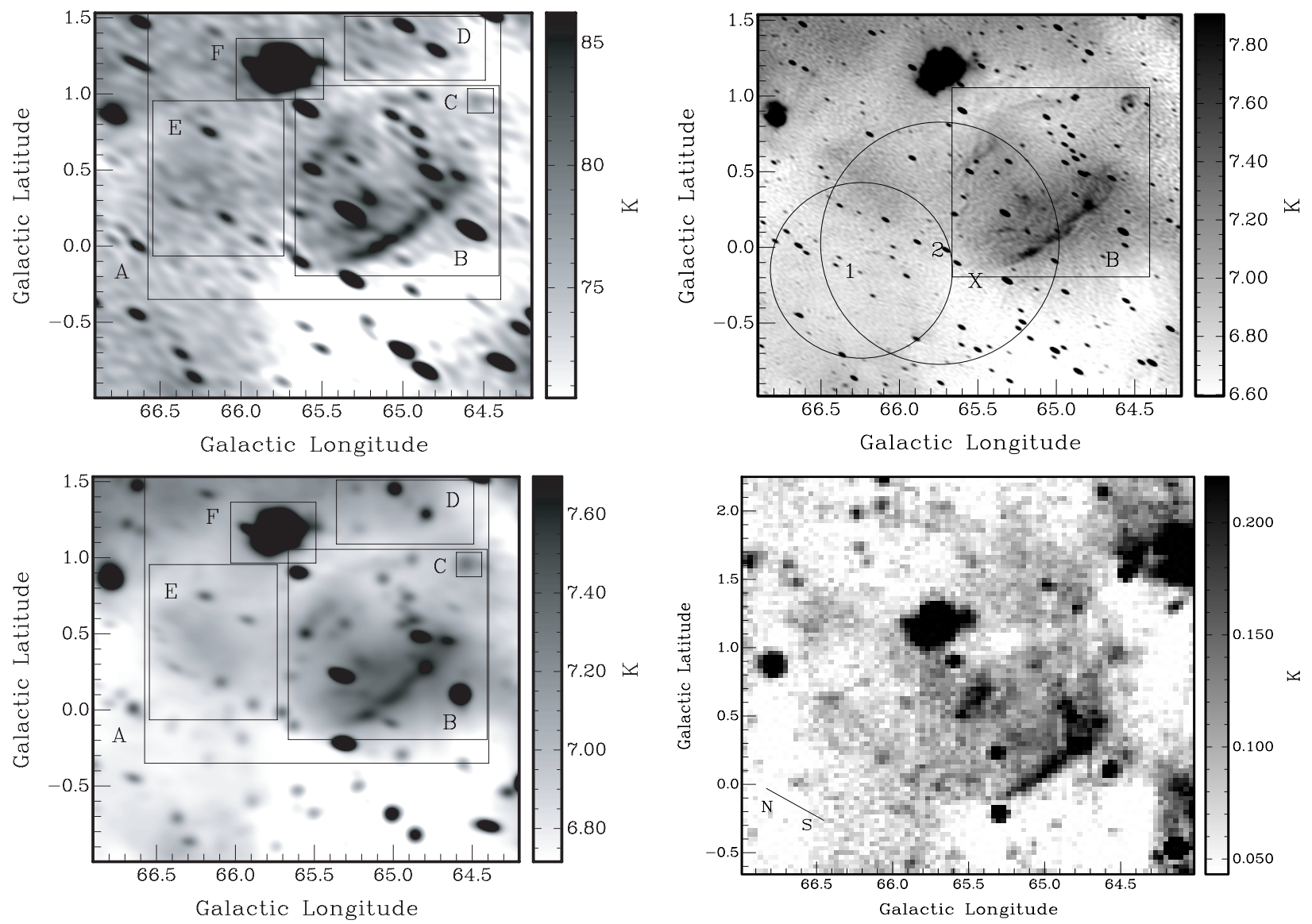

Fig. 1. The first row of images shows the CGPS maps at $408 \mathrm{MHz}$ (left) and $1420 \mathrm{MHz}$ (right). The left of the second row shows the $1420 \mathrm{MHz}$ map convolved to the same resolution as the $408 \mathrm{MHz}$ map. The right of the second row is the $2695 \mathrm{MHz}$ Effelsberg map. The 6 boxes labeled with letters A-F and used for T-T plots are shown in the left. The right of the first row shows positions and error circles of EGRET source 3EG J1958+2909 (95\% confidence level, marked by number 1) and $\gamma$-ray 2CG 065+00 (90\% confidence level, marked by number 2). Pulsar $\mathrm{J} 1957+2831$ is marked by letter X. The direction of North (N) and South (S) is marked on the lower right image.

The $408 \mathrm{MHz}$ image for G65.1+0.6 reveals more structures in comparison with Landecker's (1990) $408 \mathrm{MHz}$ image, especially, side-lobe effects which are obvious in Landecker's maps are much reduced on our image. The $1420 \mathrm{MHz}$ map shows much better detail of the fine structure in G65.1+0.6 than any previous image. More compact sources within the SNR are resolved in the $1420 \mathrm{MHz}$ map. More filament structures appear in the northern, northwestern and southwestern parts of the SNR. Figure 2 shows that a ring-shape structure inside box $\mathrm{C}$ is clearly detected near the western diffuse emission regions of the SNR. The outlines of G65.1+0.6 at $408 \mathrm{MHz}$ and $1420 \mathrm{MHz}$ are very similar.

The $3^{\circ} \times 3^{\circ} 2695 \mathrm{MHz}$ Effelsberg map appears to show a circular-shell (Radius about $1^{\circ}$ ) SNR-like emission region including the two known SNRs G65.1+0.6 and G65.7+1.2 (DA495). But the CGPS maps at both $408 \mathrm{MHz}$ and $1420 \mathrm{MHz}$ don't show this circular shell. We mark the large emission region (A) and its two inner areas (D and E) related with G65.1+0.6 in order to study the emission further.

\subsection{T-T plot spectral indices}

Bright compact sources affect the measured integrated flux densities for G65.1+0.6 and its measured spectral index. Thus we correct for the effects of compact sources. Table 1 lists properties of the 16 brightest compact sources which are detected in

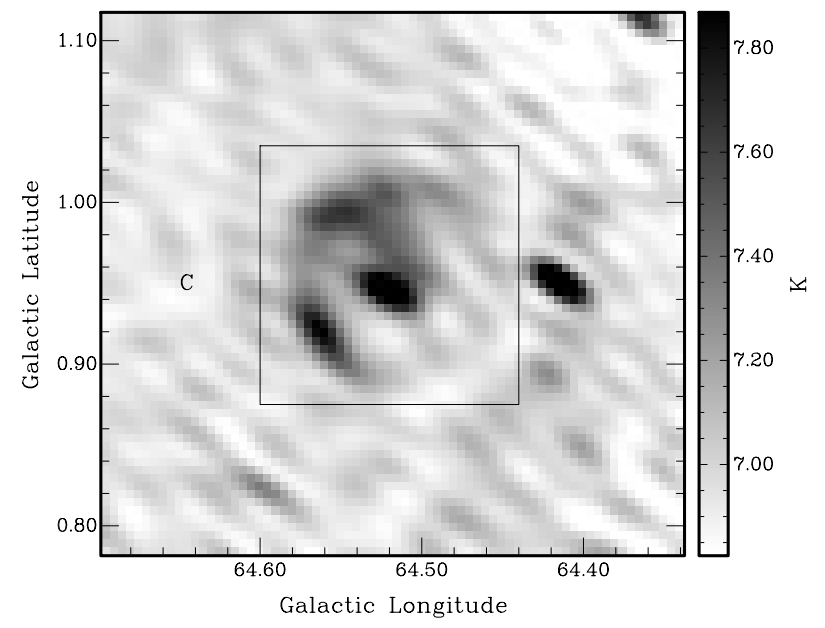

Fig. 2. Image of box C at $1420 \mathrm{MHz}$.

box A, and the first 11 of which are within G65.1+0.6 at both $408 \mathrm{MHz}$ and $1420 \mathrm{MHz}$.

First we discuss spectral indices between $408 \mathrm{MHz}$ and $1420 \mathrm{MHz}$ based on the T-T plot method (Turtle et al. 1962). The principle of the T-T plot method is that spectral indices $\left(T_{v}=T_{o} v^{-\beta}\right)$ are calculated from a fit of a linear relation to the $T_{1}-T_{2}$ values of all pixels within a given map region. $T_{1}$ is the 
Table 1. List of the 16 brightest compact sources (the first 11 sources are within G65.1+0.6) and their integrated flux densities within box A.

\begin{tabular}{cccccccc}
\hline \hline No. & RA(2000) & Dec(2000) & GLON & GLAT & $S_{408 \mathrm{MHz}}$ & $S_{1420 \mathrm{MHz}}$ & Sp. Index \\
\hline & {$[\mathrm{h} \mathrm{m} \mathrm{s}]$} & {$\left[{ }^{0}{ }^{\prime \prime}\right]$} & $\mathrm{deg}$ & $\mathrm{deg}$ & $\mathrm{mJy}$ & $\mathrm{mJy}$ & $\alpha$ \\
\hline 1 & 195346.53 & 275256.6 & 64.566 & 0.109 & $1388 \pm 42$ & $533 \pm 17$ & $0.77(0.73$ to 0.80$)$ \\
2 & 19553.77 & 283543.2 & 65.323 & 0.234 & $1044 \pm 34$ & $318 \pm 10$ & $0.95(0.92$ to 0.99$)$ \\
3 & 19533.10 & 291030.2 & 65.593 & 0.910 & $610 \pm 19$ & $261 \pm 9$ & $0.68(0.65$ to 0.72$)$ \\
4 & 195253.01 & 281743.0 & 64.819 & 0.490 & $267 \pm 20$ & $111 \pm 4$ & $0.71(0.64$ to 0.78$)$ \\
5 & 19572.74 & 283952.6 & 65.608 & -0.102 & $229 \pm 16$ & $37 \pm 3$ & $1.45(1.36$ to 1.55$)$ \\
6 & 19523.14 & 282946.9 & 64.898 & 0.750 & $191 \pm 37$ & $31 \pm 7$ & $1.46(1.12$ to 1.80$)$ \\
7 & 195611.84 & 275946.5 & 64.940 & -0.290 & $147 \pm 9$ & $61 \pm 6$ & $0.70(0.61$ to 0.81$)$ \\
8 & 195157.62 & 284148.9 & 65.060 & 0.870 & $137 \pm 30$ & $44 \pm 3$ & $0.91(0.66$ to 1.13$)$ \\
9 & 195431.32 & 285559.3 & 65.551 & 0.510 & $127 \pm 10$ & $49 \pm 3$ & $0.76(0.69$ to 0.84$)$ \\
10 & 19577.54 & 285825.4 & 65.881 & 0.044 & $94 \pm 10$ & $46 \pm 2$ & $0.58(0.49$ to 0.67$)$ \\
11 & 195651.73 & 284624.5 & 65.680 & -0.011 & $83 \pm 13$ & $60 \pm 4$ & $0.26(0.13$ to 0.41$)$ \\
\hline 12 & 195646.80 & 282133.9 & 65.317 & -0.211 & $765 \pm 25$ & $577 \pm 340$ & $0.23(-0.15$ to 0.94$)$ \\
13 & 194927.70 & 285557.2 & 64.984 & 1.461 & $290 \pm 12$ & $135 \pm 7$ & $0.62(0.57$ to 0.67$)$ \\
14 & 194939.44 & 284027.0 & 64.783 & 1.293 & $281 \pm 12$ & $100 \pm 4$ & $0.83(0.78$ to 0.87$)$ \\
15 & 19553.55 & 29366.0 & 66.184 & 0.755 & $117 \pm 6$ & $56 \pm 2$ & $0.75(0.70$ to 0.80$)$ \\
16 & 195222.96 & 29561.9 & 66.170 & 1.424 & $48 \pm 6$ & $42 \pm 1$ & $0.11(0.01$ to 0.21$)$ \\
\hline
\end{tabular}

brightness temperature of a map pixel at one frequency and $T_{2}$ is for the second frequency. The higher resolution image has been smoothed to the lower resolution for the T-T plot comparison. The brightness temperature spectral index $\beta$ is derived from the slope of the line. The error in spectral index is derived from the uncertainly in slope of the line. The flux density spectral index $\alpha$ $\left(S_{v} \propto v^{-\alpha}\right)$ is related to $\beta$ by $\beta=\alpha+2$. Spectral index refers to flux density spectral index $\alpha$ in this paper unless specifically noted otherwise.

For the T-T plot spectral index analysis, we select a single large region (A) including the two SNRs G65.1+0.6 and DA495, region $\mathrm{B}$ including $\mathrm{G} 65.1+0.6$, region $\mathrm{F}$ including DA495 alone and other 3 areas (C, D, E) around G65.1+0.6, as shown in Fig. 1. Region A yields the T-T plots shown in the upper half of Fig. 3. Region B yields the T-T plots shown in the lower half of Fig. 3.

In the T-T plot analysis, two cases are considered: using all pixels including compact sources; and excluding compact sources listed in Table 1 from the images. The compact sources are bright compared to the SNR emission. Since the compact sources have a steeper spectrum than the SNR, they are seen in the T-T plot (Fig. 3 left panel) as the steeper lines of points extending to higher $T_{\mathrm{B}}$. We completely remove regions of pixels including the compact sources from the analysis. Each region is taken to be a few beamwidths across, so that any contribution from the compact source is below $1 \%$ of the diffuse SNR emission.

Table 2 lists the results for two cases of analysis: including compact sources and removing compact sources. Visual inspection of the T-T plots confirms that the second method produces the most reliable results. The compact sources' influence on the spectral index calculation is obvious in the T-T plots of Fig. 3, and also seen in Table 2 for areas A, B, D and E. From now on we discuss spectral indices derived with compact sources removed, unless specified otherwise.

Region A includes several emission regions. The spectral index for a box F including DA495 alone is given in Table 2 $(0.50 \pm 0.01)$. From the right panels of Fig. 3 , it is obvious that the T-T plot of G65.1+0.6 is only left lower part of region A's T-T plot. The upper right part of region A's plot is from the SNR DA495.
Table 2. 408-1420 MHz T-T plot spectral indices with and without Compact Sources (CS).

\begin{tabular}{ccc}
\hline \hline Sp. Index & $\alpha$ & $\alpha$ \\
\hline Area & including CS & CS removed \\
\hline A & $0.30 \pm 0.21$ & $0.25 \pm 0.22$ \\
B & $0.43 \pm 0.21$ & $0.34 \pm 0.20$ \\
C & $0.35 \pm 0.13$ & $0.35 \pm 0.13$ \\
D & $0.59 \pm 0.13$ & $0.51 \pm 0.20$ \\
E & $0.34 \pm 0.59$ & $0.18 \pm 1.20 *$ \\
F & $0.50 \pm 0.01$ & $0.50 \pm 0.01$ \\
\hline
\end{tabular}

*Manual fitting gives a more reliable value 0.7 .

\subsection{Integrated flux densities and spectral indices}

We have derived integrated flux densities of G65.1+0.6 from the $408 \mathrm{MHz}$ and $1420 \mathrm{MHz}$ maps. Values given have diffuse background subtracted. The resulting $408 \mathrm{MHz}$ to $1420 \mathrm{MHz}$ spectral index, using flux densities without compact sources, is $0.45 \pm 0.11$. Table 3 lists the flux densities and spectral indices of G65.1+0.6 and the compact sources within G65.1+0.6. Compact sources contribute about $33 \%$ at both $408 \mathrm{MHz}$ and $25 \%$ at $1420 \mathrm{MHz}$ to the SNR's flux densities, and have a significant effect on the spectral index. It is noted that the SNR spectral index derived from integrated flux densities is consistent with the SNR spectral index $(0.34 \pm 0.20)$ derived by the T-T plot method.

We have calculated total compact source flux densities for $2695 \mathrm{MHz}$ frequencies, using the 408-1420 MHz spectral index upper and lower limits and flux densities from Table 1. The compact sources contribute $21 \%$ at $2695 \mathrm{MHz}$ to the SNR's flux densities. We have recalculated the flux density values of G65.1+0.6 at $2695 \mathrm{MHz}$ by subtracting the compact source flux density. We note that Fürst et al. (1984) gave a flux density based on the $2965 \mathrm{MHz}$ Effelsberg image with resolution $4.4 \times 4.4$ arcmin, but we obtain a new value from the Effelsberg $2695 \mathrm{MHz}$ image with a little higher resolution $4.3 \times 4.3$ arcmin: $3.3 \pm 0.5 \mathrm{Jy}$ and use this instead. We fit the resulting flux density values with a power-law to obtain spectral index. Figure 4 shows the corrected flux densities and the best-fit power-law. The best fit spectral index is 0.49 with $1 \sigma$ uncertainty range of 0.40 to 0.57 . The fit to G65.1+0.6 spectrum including compact sources flux 

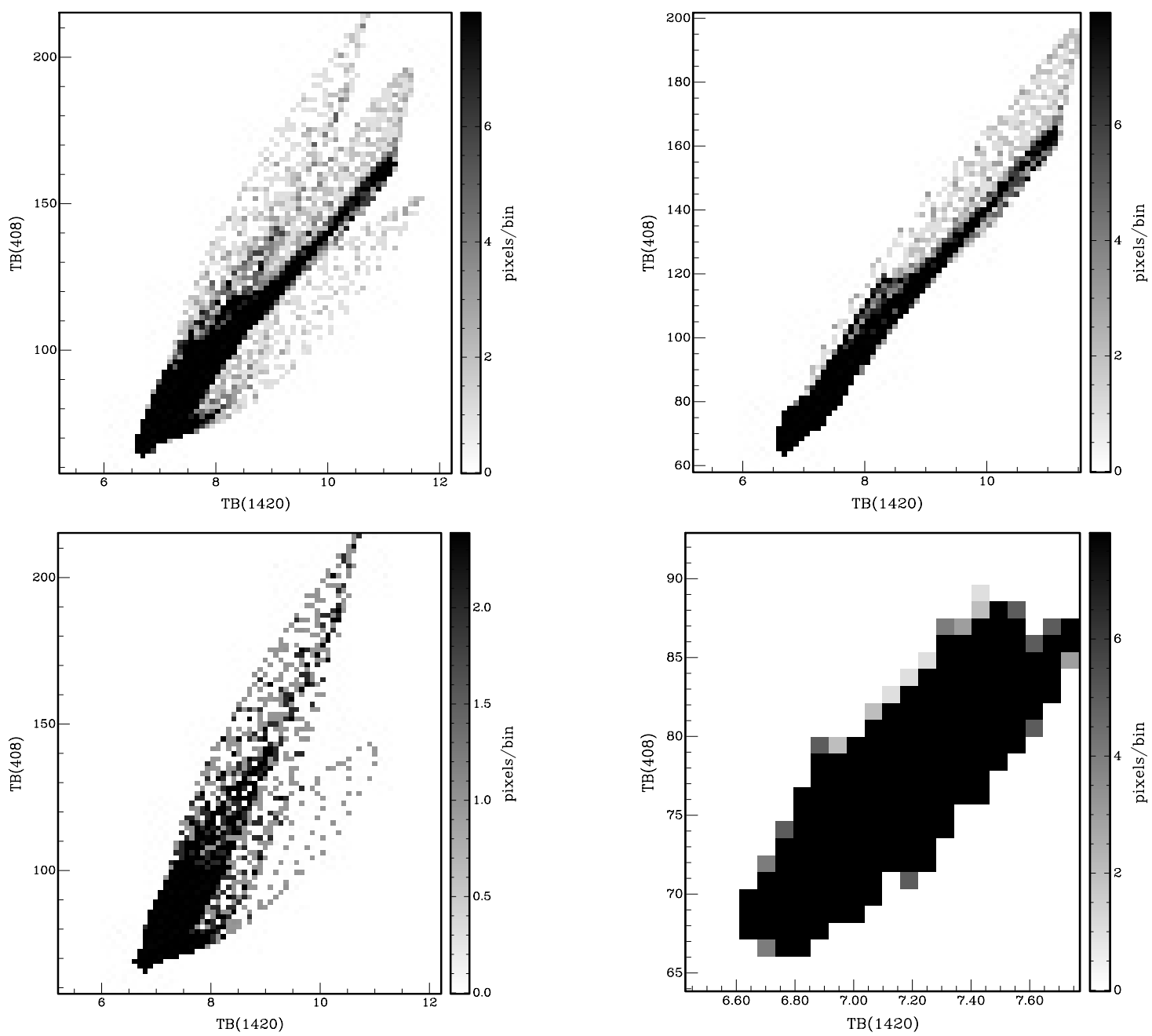

Fig. 3. 408-1420 MHz T-T plots for region A (the upper half) and region B (the lower half). The left plots for maps including compact sources; The right plots for compact sources removed from analysis.

Table 3. Integrated flux densities and $408-1420 \mathrm{MHz}$ spectral indices of G65.1+0.6 and compact sources within the SNR.

\begin{tabular}{cccc}
\hline \hline Freq. & G65.1+0.6 & CS of G65.1+0.6 & G65.1+0.6 and CS \\
\hline MHz & Jy & Jy & Jy \\
\hline 408 & $8.6 \pm 0.8$ & $4.3 \pm 0.2$ & $12.9 \pm 1.0$ \\
1420 & $4.9 \pm 0.5$ & $1.6 \pm 0.1$ & $6.5 \pm 0.6$ \\
2695 & $3.3 \pm 0.5$ & $0.9 \pm 0.1$ & $4.2 \pm 0.4$ \\
\hline$\alpha$ & $0.45 \pm 0.11$ & $0.79 \pm 0.06$ & $0.55 \pm 0.10$ \\
\hline
\end{tabular}

density gives $\alpha=0.58$. The steeper $\alpha$ is expected since compact source has the steeper spectrum than SNR (see Table 3).

\subsection{HI emission}

We have searched the CGPS radial velocity range for features in the HI which might relate to the morphology of G65.1+0.6. There is emission which is coincident with the boundary of G65.1+0.6 in the velocity range -20 to $-26 \mathrm{~km} \mathrm{~s}^{-1}$, and only in this velocity range. The spatial relation with the edge of G65.1+0.6 indicates that the HI is associated with the SNR. These HI observations show a good association of HI features with G65.1+0.6, similar to other accepted HI associations with SNRs and with similar HI velocity ranges $\left(\sim 10 \mathrm{~km} \mathrm{~s}^{-1}\right)$, e.g. HI associated with G126.2+1.6 (Tian \& Leahy 2006), with

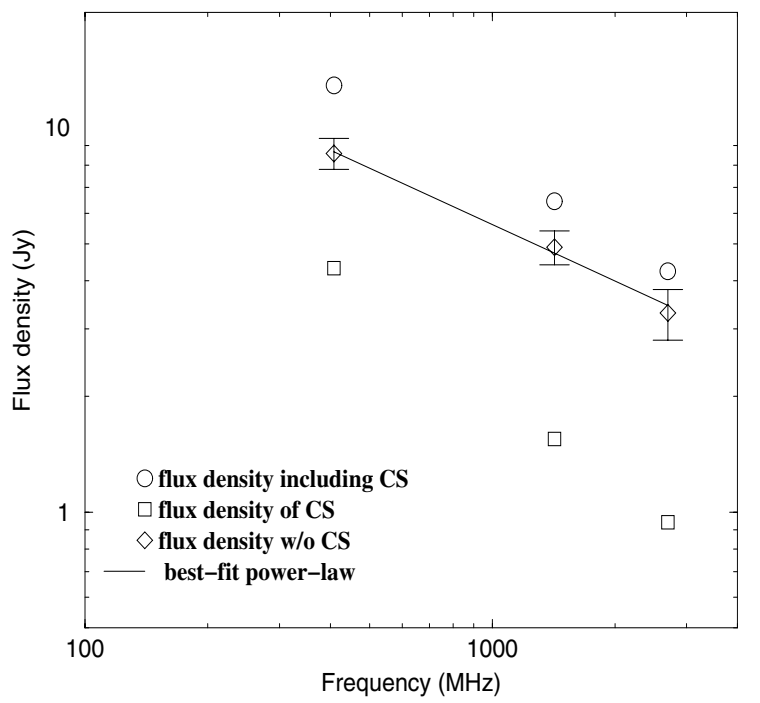

Fig. 4. Radio spectrum of G65.1+0.6 with and without Compact Sources (CS). The power law model has a best-fit spectral index of 0.49 with $1 \sigma$ uncertainty range of 0.40 to 0.57 .

G127.1+0.6 (Leahy \& Tian 2006) and with DA530 (Landecker et al. 1999). Figure 5 shows maps of HI emission in twelve 

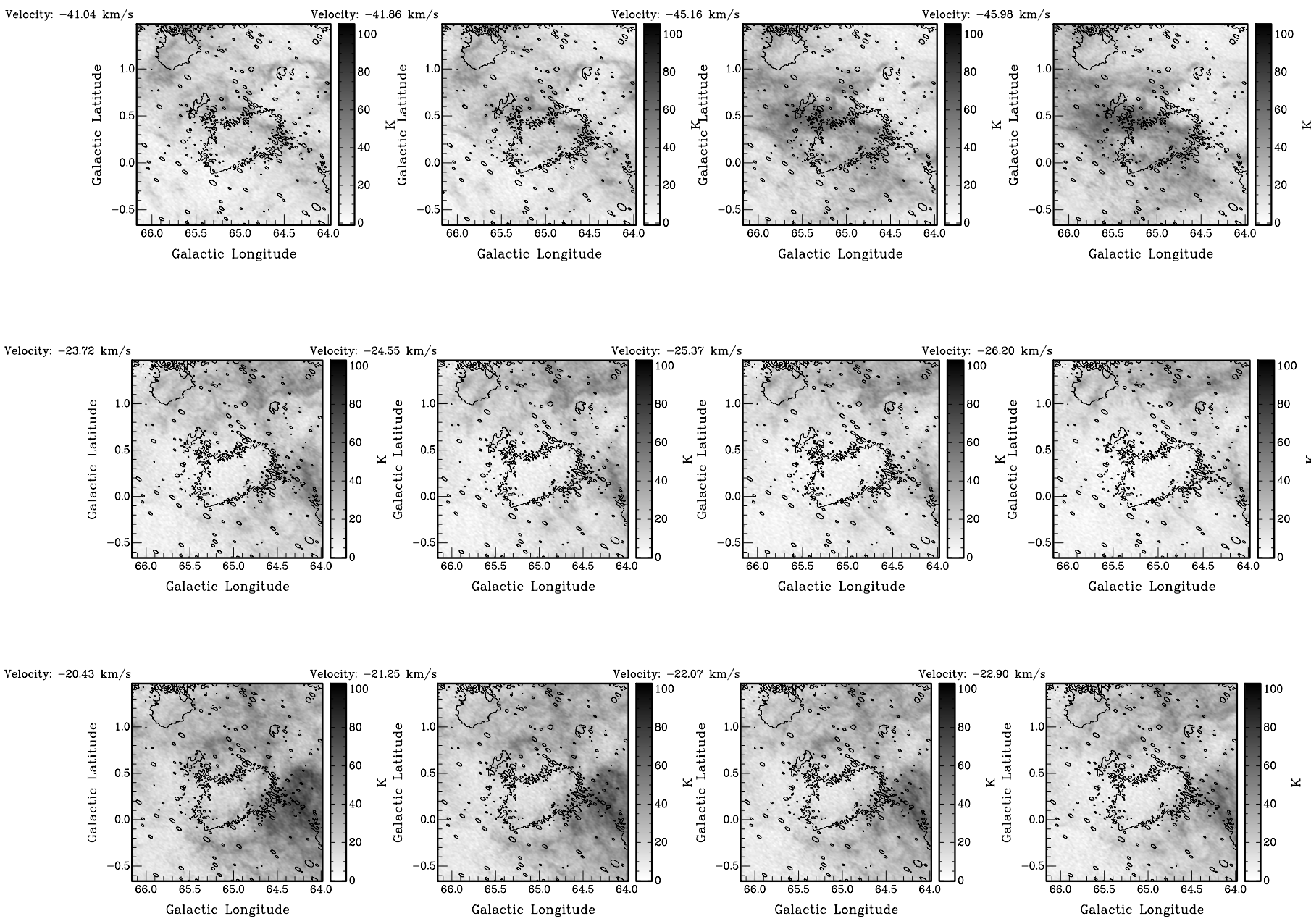

Fig. 5. HI emission in the field centered on G65.1+0.6 from -20 to $-26 \mathrm{~km} \mathrm{~s}^{-1}$ and -41 to $-46 \mathrm{~km} \mathrm{~s}^{-1}$. The radial velocity of each map is indicated at its top left corner. The outline of G65.1+0.6 in $1420 \mathrm{MHz}$ continuum emission is indicated by the contour at $7.2 \mathrm{~K} T_{\mathrm{B}}$.

channels. Each map has superimposed on it contours of continuum emission at $1420 \mathrm{MHz}$ chosen to show G65.1+0.6.

\subsection{EGRET source, $\gamma$-ray and pulsar nearby the SNR}

The upper right of Fig. 1 shows positions of the EGRET source 3EG J1958+2909 detected by Hartman et al. (1999), the TeV $\gamma$-ray source 2CG $065+00$ detected by Alexandreas et al. (1991) and the PSR J1957+2831 discovered by Lorimer (1998). The EGRET and $\gamma$-ray sources overlap each other and the SNR G65.1+0.6. The EGRET and TeV sources are likely the same source with position inconsistent with the pulsar's position and are likely not associated with the SNR. The pulsar is nearby at the eastern boundary of the SNR G65.1+0.6.

\section{Discussion}

\subsection{The distance and age to G65.1+0.6}

Since the velocity is negative $\left(-20\right.$ to $-26 \mathrm{~km} \mathrm{~s}^{-1}$ ) this implies it is outside the solar circle and at $l=65.1^{\circ}$, is very far away. We use circular galactic rotation velocity $V_{R}=V_{0}=220 \mathrm{~km} \mathrm{~s}^{-1}$. Then $R_{0} / R=0.89$ for $-22 \mathrm{~km} \mathrm{~s}^{-1}, 0.87$ for $-26 \mathrm{~km} \mathrm{~s}^{-1}$ and 0.90 for $-20 \mathrm{~km} \mathrm{~s}^{-1}$. Then we use $R_{0}=8.5 \mathrm{kpc}$, so $d=9.2 \mathrm{kpc}$ $\left(-22 \mathrm{~km} \mathrm{~s}^{-1}\right), 9.0 \mathrm{kpc}\left(-20 \mathrm{~km} \mathrm{~s}^{-1}\right)$ and $9.6 \mathrm{kpc}\left(-26 \mathrm{~km} \mathrm{~s}^{-1}\right)$.
Non-circular motions in the HI feature increase the distance uncertainty, e.g. $\pm 5 \mathrm{~km} \mathrm{~s}^{-1}$, increases the distance range to $8.7-10.1 \mathrm{kpc}$. This means G65.1+0.6 is very large (angular diameters $90^{\prime}$ by $51^{\prime}$, average diameter $70^{\prime}$ ): yields radius $93.5 \mathrm{pc}$ $(d=9.2 \mathrm{kpc})$.

The SNR is of low surface brightness and large radius, so probably exploded in a low density part of the ISM. A Sedov model (Cox 1972) gives $t=14 \times 10^{4} \mathrm{yr}$ if $n_{0}=0.01 \mathrm{~cm}^{-3}$, or $t=$ $4.4 \times 10^{4} \mathrm{yr}$ if $n_{0}=0.001 \mathrm{~cm}^{-3}$. The radius at which cooling affects the dynamics of the SNR is $68 \mathrm{pc}$ if $n_{0}=0.01 \mathrm{~cm}^{-3}$, or $127 \mathrm{pc}$ if $n_{0}=0.001 \mathrm{~cm}^{-3}$. So it is possible that the SNR is still in the Sedov phase or has already entered the cooling phase depending on the density $n_{0}$. The shock temperature is $8 \times 10^{5} \mathrm{~K}$ if $n_{0}=0.01$ and $8 \times 10^{6} \mathrm{~K}$ if $n_{0}=0.001$. Since the density is low it would be faint in X-rays, and since it is quite distant it would be absorbed in X-rays by the intervening ISM. The nondetection in the Rosat All-sky Survey of G65.1+0.6 is consistent with a large distance to G65.1+0.6.

\subsection{Possible association of the SNR G65.1+0.6 and the pulsar J1957+2831}

Lorimer et al. (1998) discovered PSR J1957+2831 which is located at the eastern edge of G65.1+0.6, and commented that 
the pulsar is not likely to be associated with G65.1+0.6 due to the large dispersion measure $(\mathrm{DM})$ distance $(7.0 \pm 2.3 \mathrm{kpc})$ and old characteristic age (1.6 Myr) of the pulsar, and their statistical grounds of pulsar-SNR pairs in the case of absence of G65.1+0.6's distance and age parameters. But Lorimer et al. (1998) also admitted a possibility that the PSR J1957+2831 may indeed be younger than its characteristic age because the pulsar has a spectral index typical for other young pulsars. If we assume that the pulsar birth-place is at geometrical centre of G65.1+0.6, and moves away toward east of the SNR at typical velocity of $500 \mathrm{~km} \mathrm{~s}^{-1}$ (Lyne et al. 1994), the kinematic age of the pulsar approximately is $18 \times 10^{4} \mathrm{yr}$ and consistent with the estimated SNR's above age. Although previous studies (Tian \& Leahy 2004; Mereghetti et al. 2002; Kaspi et al. 2001) have suggested that pulsar characteristic ages can be poor age estimators for young pulsars, the pulsar J1957+2831 is not young. The pulsar's DM distance $(7.0 \pm 2.3 \mathrm{kpc})$ and kinematic age agree with G65.1+0.6, and its characteristic disagrees with the age of the SNR, so it is possible that the pulsar is associated with the SNR.

\subsection{New supernova remnant G64.5+0.9?}

The $2695 \mathrm{MHz}$ Effelsberg map appears to show a circular-shell SNR-like emission region most of which is included in region A shown in Fig. 1. The 408-1420 MHz T-T plot spectral index of areas C, D, E in Table 3 shows they are probably all nonthermal emission regions (each is about $2 \sigma$ different from thermal spectral index). This supports the possibility that there is a large diffuse SNR including the two known SNRs. But the HI images don't show any association of HI features with the large circular shell. Thus current evidence is inconclusive.

The ring-like structure in box $\mathrm{C}$ detected in the $1420 \mathrm{MHz}$ image (Fig. 2) may be part of the western edge of G65.1+0.6. However, the ring has a good association with HI features in the velocity range -41 to $-46 \mathrm{~km} \mathrm{~s}^{-1}$ as shown in Fig. 5. The HI velocity corresponds to a distance of $11 \mathrm{kpc}$ $\left(-43 \mathrm{~km} \mathrm{~s}^{-1}\right)$. Now we name the emission structure as a possible new SNR G64.5+0.9 which certainly needs to be confirmed by future high resolution and sensitivity observations.

\section{Conclusion}

Using the $408 \mathrm{MHz}$ and $1420 \mathrm{MHz}$ continuum emission and the HI-line emission data of the CGPS, physical parameters of G65.1+0.6: flux densities, spectral index, distance and age are either corrected or first obtained. Based on distance and age of both the PSR J1957+2831 and the SNR G65.1+0.6, we conclude that the pulsar and the SNR are possibly associated. Our analysis of G65.1+0.6 shows that the integrated flux density based spectral index between $1420 \mathrm{MHz}$ and $408 \mathrm{MHz}(0.45 \pm 0.11)$, $\mathrm{T}-\mathrm{T}$ plot spectral index $(0.34 \pm 0.20)$ and the fit spectral index (0.49) are consistent. The nearby SNR DA495 has a T-T plot spectral index of $0.50 \pm 0.01$. A possible SNR G64.5+0.9 is suggested for its likely nonthermal emission feature, shell-type structure and associating with HI emission features.

Acknowledgements. We acknowledge support from the Natural Sciences and Engineering Research Council of Canada. We thank Dr. R. Kothes at the DRAO provided technical help on the CGPS data processing. The DRAO is operated as a national facility by the National Research Council of Canada. The Canadian Galactic Plane Survey is a Canadian project with international partners.

\section{References}

Alexandreas, D. E., Berley, D., Biller, S., et al. 1991, ApJ, 383, 53 Cox, D. 1972, ApJ, 178, 159

Fürst, E., Reich, W., \& Steube, R. 1984, A\&A, 133, 11

Fürst, E., Reich, W., Reich, P., \& Reif, K. 1990, A\&AS, 85, 691

Hartman, R. C., Bertsch, D. L., Bloom, S. D., et al. 1999, ApJS, 123, 79

Haslam, C. G. T., Salter, C. J., Stoffel, H., \& Wilson, W. W. 1982, A\&AS, 47, 1 Higgs, L. A., \& Tapping, K. F. 2000, AJ, 120, 2471

Kaspi, V. M., Roberts, M. E., Vasisht, G., et al. 2001, ApJ, 560, 371

Landecker, T. L., Clutton-Brock, M., \& Purton, C. R. 1990, A\&A, 232, 207

Landecker, T. L., Routledge, D., Reynolds, S. P., et al. 1999, ApJ, 527, 866

Leahy, D. A., \& Tian, W. W. 2006, A\&A, 451, 251

Lorimer, D. R., Lyne, A. G., \& Camilo, F. 1998, 331, 1002

Mereghetti, S., de Luca, A., Caraveo, P. A., et al. 2002, ApJ, 581, 1280

Reich, W., Reich, P., \& Fürst, E. 1990, A\&AS, 83, 539

Reich, W., Reich, P., \& Fürst, E. 1997, A\&AS, 126, 413

Swanenburg, B. N., Bennett, K., Bignami, G. F., et al. 1981, ApJ, 243, L69

Taylor, A. R., Gibson, S. J., Peracaula, M., et al. 2003, AJ, 125, 3145

Tian, W. W., \& Leahy, D. A. 2006, A\&A, 447, 205

Tian, W. W., \& Leahy, D. A. 2005, A\&A, 436, 187

Tian, W. W., \& Leahy, D. A. 2004, Progr. Astron., 22, 308

Turtle, A. J., Pugh, J. F., Kenderdine, S., \& Pauliny-Toth, I. I. K. 1962, MNRAS, 124, 297 